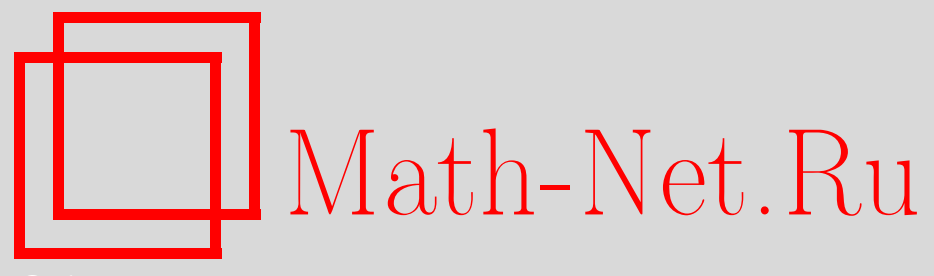

О. И. Мохов, Римановы инварианты полупростых нелокально-бигамильтоновых систем гидродинамического типа и согласованные метрики, УМH, 2010, том 65, выпуск 6, 189-190

DOI: https://doi.org/10.4213/rm9391

Использование Общероссийского математического портала Math-Net.Ru подразумевает, что вы прочитали и согласны с пользовательским соглашением http://www . mathnet.ru/rus/agreement

Параметры загрузки:

IP: 34.229 .108 .108

26 апреля 2023 г., 13:50:00

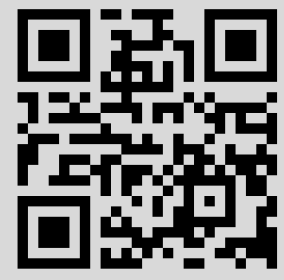




\section{Римановы инварианты полупростых нелокально-бигамильтоновых систем гидродинамического типа и согласованные метрики}

\section{О. И. Мохов}

В данной заметке доказана диагонализуемость произвольной неособой (полупростой) нелокально-бигамильтоновой системы гидродинамического типа и для каждой такой системы построен полный набор римановых инвариантов, полностью определяемый метриками бигамильтоновой структуры.

Рассмотрим системы гидродинамического типа, которые являются бигамильтоновыми относительно двух линейно независимых согласованных невырожденных нелокальных скобок Пуассона гидродинамического типа, т. е.

$$
\begin{gathered}
u_{t}^{i}=V_{j}^{i}(u) u_{x}^{j}=\left\{u^{i}(x), H_{1}\right\}_{1}=\left\{u^{i}(x), H_{2}\right\}_{2}, \quad 1 \leqslant i, j \leqslant N, \\
H_{\alpha}=\int h_{\alpha}(u(x)) d x, \quad\{I, J\}_{\alpha}=\int \frac{\delta I}{\delta u^{i}(x)} P_{\alpha}^{i j} \frac{\delta J}{\delta u^{j}(x)} d x, \\
P_{\alpha}^{i j}=g_{\alpha}^{i j}(u(x)) \frac{d}{d x}+b_{\alpha, k}^{i j}(u(x)) u_{x}^{k} \\
+\sum_{m, n=1}^{L} \mu_{\alpha}^{m n}\left(w_{\alpha, m}\right)_{k}^{i}(u(x)) u_{x}^{k}\left(\frac{d}{d x}\right)^{-1} \circ\left(w_{\alpha, n}\right)_{s}^{j}(u(x)) u_{x}^{s}, \quad \alpha=1,2 .
\end{gathered}
$$

(Скобки Пуассона вида (2), (3) введены и исследованы в [1].) Нелокально-бигамильтонова система гидродинамического типа (1)-(3) называется неособой (или полупроcтой), если пара метрик $g_{1}^{i j}(u)$ и $g_{2}^{i j}(u)$ является неособой (полупростой), т. е. собственные значения этой пары метрик (корни уравнения $\operatorname{det}\left(g_{1}^{i j}(u)-\lambda g_{2}^{i j}(u)\right)=0$ ) различны.

Теорема. Для произвольной полупростой нелокально-бигамильтоновой системы гидродинамического типа (1)-(3) существуют локальные координать, в которых афбинор $v_{j}^{i}(u)=g_{1}^{i s}(u) g_{2, s j}(u)$, задаваемый метриками бигамильтоновой структуры, является диагональным; эти локальные координаты всегда являются римановыми инвариантами рассматриваемой полупростой системы (1)-(3), т.е. матрица (аффинор) $V_{j}^{i}(u)$ этой системы гидродинамического типа является диагональной в этих локальных координатах. Более того, все осталъные связанные с данной системой матричные дифференциалъно-геометрические объекты, а именно, метри$\kappa и g_{1}^{i j}(u)$ и $g_{2}^{i j}(u)$ и аффиноры $\left(w_{1, n}\right)_{j}^{i}(u) u\left(w_{2, n}\right)_{j}^{i}(u)$ нелокальной бигамильтоновой структуры этой системы, также всегда являются диагональными в этих локальных координатах.

В [2] автором было доказано, что для любой пары линейно независимых согласованных невырожденных нелокальных скобок Пуассона гидродинамического типа $(2),(3)$ метрики $g_{1}^{i j}(u)$ и $g_{2}^{i j}(u)$ являются согласованными [2], [3], т. е. для любой линейной комбинации этих метрик $g^{i j}(u)=\lambda_{1} g_{1}^{i j}(u)+\lambda_{2} g_{2}^{i j}(u)$, где $\lambda_{1}$ и $\lambda_{2}$ - произвольные константы, коэффициенты соответствующих связностей Леви-Чивиты и компоненты соответствующих тензоров римановой кривизны связаны тем же самым линейным соотношением: $\Gamma_{k}^{i j}(u)=\lambda_{1} \Gamma_{1, k}^{i j}(u)+\lambda_{2} \Gamma_{2, k}^{i j}(u), R_{k l}^{i j}(u)=\lambda_{1} R_{1, k l}^{i j}(u)+\lambda_{2} R_{2, k l}^{i j}(u)$. В [2], [3] автором было доказано, что указанное выше соотношение на связности Леви-Чивиты

Работа выполнена при поддержке РФФИ (гранты № 08-01-00054 и № 09-01-12148-офи-м) и программы "Ведущие научные школы" (грант НШ-1824.2008.1). 
для произвольной пары метрик выполняется тогда и только тогда, когда тензор Нейенхейса аффинора $v_{j}^{i}(u)=g_{1}^{i s}(u) g_{2, s j}(u)$, задаваемого метриками, тождественно равен нулю (почти согласованные метрики, см. [2], [3]). Для полупростой пары метрик $g_{1}^{i j}(u)$ и $g_{2}^{i j}(u)$ все собственные значения аффинора $v_{j}^{i}(u)=g_{1}^{i s}(u) g_{2, s j}(u)$ различны (они совпадают с собственными значениями этой пары метрик), а в этом случае по теореме Нейенхейса [4] из обнуления тензора Нейенхейса аффинора следует, что существуют локальные координаты, в которых этот аффинор является диагональным. Таким образом, для произвольной полупростой нелокально-бигамильтоновой системы гидродинамического типа (1)-(3) существуют локальные координаты, в которых аффинор $v_{j}^{i}(u)=g_{1}^{i s}(u) g_{2, s j}(u)$ является диагональным. Рассмотрим любые локальные координаты, в которых аффинор $v_{j}^{i}(u)=g_{1}^{i s}(u) g_{2, s j}(u)$ рассматриваемой полупростой системы (1)-(3) является диагональным. Так как все собственные значения этого аффинора различны, то из обнуления тензора Нейенхейса аффинора следует, что он имеет специальный диагональный вид $v_{j}^{i}(u)=f^{i}\left(u^{i}\right) \delta_{j}^{i}$ (разделение переменных). Если для полупростой пары метрик аффинор $v_{j}^{i}(u)=g_{1}^{i s}(u) g_{2, s j}(u)$ диагонален, то обе метрики также диагональны, более того, в нашем случае в рассматриваемых координатах эта пара метрик обязана иметь следующий специальный диагональный вид: $g_{2}^{i j}(u)=g^{i}(u) \delta^{i j}, g_{1}^{i j}(u)=f^{i}\left(u^{i}\right) g^{i}(u) \delta^{i j}$. В [2] доказано, что любые пары метрик такого вида всегда согласованы, т. е. выполняется и соответствующее соотношение на тензоры римановой кривизны. Для произвольной системы гидродинамического типа, гамильтоновой относительно невырожденной нелокальной скобки Пуассона гидродинамического типа (2), (3), всегда выполнены следующие важные соотношения: $g_{i s}(u) V_{j}^{s}(u)=g_{j s}(u) V_{i}^{s}(u), \nabla_{j} V_{k}^{i}(u)=\nabla_{k} V_{j}^{i}(u)$. В случаях локальной скобки Дубровина-Новикова [5] и нелокальной скобки Мохова-Ферапонтова [6] эти соотношения являются необходимыми и достаточными для соответствующей гамильтоновости системы (см. [7] для локального случая и [6] для нелокального). Из этих соотношений следует, что аффинор $V_{j}^{i}(u)$ обязан быть диагональным в рассматриваемых координатах. Диагонализуемость системы в локальном случае (см., например, [8]) сразу следует из этих соотношений и теории согласованных плоских метрик (см. [2], [3], [9]). Диагональность аффиноров $\left(w_{1, n}\right)_{j}^{i}(u)$ и $\left(w_{2, n}\right)_{j}^{i}(u)$ нелокальной бигамильтоновой структуры в рассматриваемых координатах следует из условий согласованности для невырожденных нелокальных скобок Пуассона гидродинамического типа (2), (3) (см. [2], [10]).

\section{Список литературы}

[1] Е. В. Ферапонтов, Функи. анализ и его прил., 25:3 (1991), 37-49. [2] О. И. Мохов, Функи. анализ и его прил., 35:2 (2001), 24-36. [3] О.И. Мохов, УМН, 55:4 (2000), 217-218. [4] A. Nijenhuis, Indag. Math., 13:2 (1951), 200-212. [5] Б. А. Дубровин, С. П. Новиков, Докл. АН СССР, 270:4 (1983), 781-785. [6] О. И. Мохов, Е. В. Ферапонтов, УМН, 45:3 (1990), 191-192. [7] С. П. Царев, Изв. АН СССР. Сер. матем., 54:5 (1990), 1048-1068. [8] B. Dubrovin, S.-Q. Liu, Y. Zhang, Comm. Pure Appl. Math., 59:4 (2006), 559-615. [9] O. I. Mokhov, J. Appl. Math., 2:7 (2002), 337-370. [10] О. И. Мохов, ТМФ, 138:2 (2004), 283-296, arXiv: math/0202036.

О. И. Мохов (О.I. Mokhov)

Московский государственный университет им. М. В. Ломоносова

E-mail: mokhov@mi.ras.ru, mokhov@landau.ac.ru
Представлено В. М. Бухштабером Принято редколлегией 22.10 .2010 